\title{
Application of colon capsule endoscopy (CCE) to evaluate the whole gastrointestinal tract: a comparative study of single-camera and dual-camera analysis
}

\author{
This article was published in the following Dove Press journal: \\ Clinical and Experimental Gastroenterology \\ 17 September 2013 \\ Number of times this article has been viewed
}

José María Remes-Troche'

Victoria Alejandra Jiménez-

García $^{2}$

Josefa María García-Montes ${ }^{2}$

Pedro Hergueta-Delgado ${ }^{2}$

Federico Roesch-Dietlen'

Juan Manuel Herrerías-

Gutiérrez ${ }^{2}$

'Digestive Physiology and Motility Lab, Medical Biological Research Institute,

Universidad Veracruzana, Veracruz,

México; ${ }^{2}$ Gastroenterology Service,

Virgen Macarena University Hospital, Seville, Spain
Correspondence: Juan Manuel HerreríasGutierrez

Gastroenterology Service,Virgen Macarena University Hospital,

Avda Dr. Fedriani, s/n4107I Sevilla, Spain

Tel +3495 5008801

Fax +3495 5008805

Email jmhg@us.es
Background and study aims: Colon capsule endoscopy (CCE) was developed for the evaluation of colorectal pathology. In this study, our aim was to assess if a dual-camera analysis using CCE allows better evaluation of the whole gastrointestinal (GI) tract compared to a single-camera analysis.

Patients and methods: We included 21 patients ( 12 males, mean age 56.20 years) submitted for a CCE examination. After standard colon preparation, the colon capsule endoscope (PillCam Colon ${ }^{\mathrm{TM}}$ ) was swallowed after reinitiation from its "sleep" mode. Four physicians performed the analysis: two reviewed both video streams at the same time (dual-camera analysis); one analyzed images from one side of the device ("camera 1"); and the other reviewed the opposite side ("camera 2"). We compared numbers of findings from different parts of the entire GI tract and level of agreement among reviewers.

Results: A complete evaluation of the GI tract was possible in all patients. Dual-camera analysis provided $16 \%$ and 5\% more findings compared to camera 1 and camera 2 analysis, respectively. Overall agreement was $62.7 \%$ (kappa $=0.44,95 \%$ CI: $0.373-0.510)$. Esophageal $(\mathrm{kappa}=0.611)$ and colorectal $(\mathrm{kappa}=0.595)$ findings had a good level of agreement, while small bowel $(\mathrm{kappa}=0.405)$ showed moderate agreement.

Conclusion: The use of dual-camera analysis with CCE for the evaluation of the GI tract is feasible and detects more abnormalities when compared with single-camera analysis.

Keywords: capsule endoscopy, colon, gastrointestinal tract, small bowel

\section{Introduction}

Capsule endoscopy (CE) has been established as an extremely useful diagnostic tool for the evaluation of obscure gastrointestinal bleeding (OGIB) and other smallbowel conditions. ${ }^{1,2}$ Currently, $\mathrm{CE}$ is approved in adult and pediatric patients older than 10 years for evaluation of OGIB, ${ }^{3,4}$ celiac disease, ${ }^{5}$ polyposis syndromes, ${ }^{6,7}$ and small-bowel abnormalities detected in imaging studies. ${ }^{8-10}$

This technology has evolved over the last decade, and new and better devices are available, such as capsules with two lenses and cameras and those capable of capturing more frames per second. ${ }^{2,11}$ The PillCam ESO ${ }^{\text {TM }}$ esophageal capsule (Given Imaging, Yoqneam, Israel), for example, has two cameras, each with the capability of taking images at seven frames per second. ${ }^{12,13}$ This device has been approved for screening and follow-up of esophageal varices and screening for Barrett's esophagus. ${ }^{12,14,15}$ More recently, a colon capsule endoscope (PillCam Colon ${ }^{\mathrm{TM}}$; Given Imaging) has been 
developed. ${ }^{16}$ The colon capsule is similar to the conventional capsule, but has two cameras that are able to record video images from both ends. ${ }^{17}$ The device measures $31 \times 11 \mathrm{~mm}$ and acquires images at a rate of four frames per second. The preprogrammed "sleep" mode allows recording of images from the esophagus and stomach for 3 minutes, after which the capsule switches to sleep mode for 1 hour 45 minutes. During this period, the capsule is likely to transit most of the small bowel; thus, by the time the capsule "wakes up" from its sleep period, it will have reached approximately the terminal ileum. The preprogrammed sleep mode saves battery, thereby allowing an almost complete evaluation of the colon.

Several studies have pointed out the effectiveness of the PillCam Colon ${ }^{\mathrm{TM}}$ capsule endoscope compared to colonoscopy for the study of colon diseases. ${ }^{18-20}$ The high-priority objective of colon capsule endoscopy (CCE) is screening for colorectal cancer in at-risk populations. ${ }^{21}$ CCE can be performed when there is a contraindication for colonoscopy, and it is suitable for patients who are unwilling to undergo colonoscopy or to complete a failed colonoscopy. It can also be used for follow-up of conditions different to adenomas or polyps, such as ulcerative colitis (UC). ${ }^{22}$

Although CCE was developed for evaluation of the colon, this dual device can also be used to assess the entire GI tract if the patient takes the capsule after early activation and as soon as the capsule recovers from its sleep mode. In a previous study, Almeida et $\mathrm{al}^{23}$ performed a study in which the colon capsule was given after the device woke up from sleep mode to ten patients for small-bowel evaluation. In this study, the authors found that enteroscopy with a doublecamera device allowed the detection of more findings and increased diagnostic accuracy when compared to a singlecamera analysis. $^{23}$

In our study, our aim was to determine if the use of CCE and a dual-camera analysis allows a better evaluation of the entire gastrointestinal (GI) tract when compared to a singlecamera analysis. In addition, we also analyzed the level of agreement between double-camera and single-camera analysis.

\section{Patients and methods}

\section{Patients}

This prospective, exploratory, and pilot study was conducted between September 2007 and October 2009 at the Hospital Virgen de la Macarena, Seville, Spain. Twenty-one (12 male, mean age 56.20 years, range 23-97 years) consecutive
Hispanic patients submitted for a PillCam Colon ${ }^{\mathrm{TM}}$ examination were included. These patients were submitted for CCE for: screening to rule out organic diseases $(n=10)$; followup of inflammatory bowel disease ([IBD] eg, Crohn's or UC history $[\mathrm{n}=5])$; chronic anemia $(\mathrm{n}=3)$; chronic abdominal pain $(\mathrm{n}=3)$; and chronic diarrhea $(\mathrm{n}=1)$. Subjects were invited to participate, and those who agreed gave their written informed consent for the procedure and the protocol analysis. This protocol was approved by the internal ethics committee of the Hospital Virgen de la Macarena.

\section{Study protocol}

\section{Small-bowel and colon preparation}

All patients underwent a 48-hour standard colon preparation for the colon capsule procedure previously described by Herrerías-Gutiérrez et al. ${ }^{17}$ Forty-eight hours before the study, the patients were put on a low-fiber diet and were instructed to drink ten glasses of water during the day. Four pills of Pursennid (12 mg sennosides A and B) were prescribed to be taken at night. The day before the test, a clear liquid diet was prescribed (water, apple juice, tea or black coffee, transparent carbonated fluids, ice cream or jelly without red or violet color, chewing gum), then the patients received $1 \mathrm{~L}$ polyethylene glycol solution between $7 \mathrm{pm}$ and $9 \mathrm{pm}$ and another liter of this solution between 7 am and 8.30 am on the day of the examination. Before (at $9.15 \mathrm{am}$ ) and 15 minutes after swallowing the capsule, the patients received $20 \mathrm{mg}$ of domperidone. Two hours later, after confirming the capsule's exit from the stomach with the real-time viewing system (Rapid Access Real Time; Given Imaging); the patient received $30 \mathrm{~mL}$ sodium phosphate solution (Fleet Phospho Soda; Casen-Fleet Laboratories, Utebo, Spain) as a booster dose. A second booster dose of $30 \mathrm{~mL}$ sodium phosphate solution was administered 3 hours after the first one if the capsule was not excreted in this period. If necessary, a bisacodyl suppository (Dulcolax; Boehringer Ingelheim, Ingelheim am Rhein, Germany) was used. Smallbowel and colon cleanliness were assessed using a grading scale, previously reported by Herrerías-Gutiérrez et al, with four grades: very good, good, fair, and poor. ${ }^{17}$

\section{PillCam Colon ${ }^{\mathrm{TM}}$ CCE device}

The PillCam Colon ${ }^{\mathrm{TM}}$ capsule used in this study is the conventional capsule $(31 \times 11 \mathrm{~mm})$ with two cameras that enable the device to acquire images from opposite directions with a wide coverage area, acquiring pictures at a rate of four frames per second. This device has a 10-hour battery life. After 3 minutes of running, the capsule turns off for a period of 1 
hour 45 minutes and then "wakes up" and restarts the transmission of images. For this protocol, we activated the PillCam prior to the procedure; the patient swallowed the capsule after the sleep period in order that images could be obtained from the whole GI tract.

\section{Data interpretation and analysis}

Four different physicians with experience in PillCam examinations interpreted the recorded data. After ingestion of the capsule, the images were read at a maximum speed of eight frames per second. Since we wanted to analyze the entire GI tract, all pathological findings, from the mouth to the rectum, were considered. When CCE is performed, the Rapid Viewer Software $^{\mathrm{TM}}$ (v 6; Given Imaging) allows simultaneous analysis of both cameras (dual-camera analysis) or single-camera analysis by deactivation of one of the two streams. In order to achieve a complete and more accurate evaluation and analysis, two physicians (PHD and JMHG) observed both video streams at the same time (dual-camera analysis). One physician (VAJG) saw only the images taken from one side of the device (conventionally named "camera 1"), and the other (JMRT) saw only the images from the opposite side ("camera 2"). The physicians were blinded to each other's results to avoid bias.

\section{Statistical analysis}

The physicians counted the total number of findings and described these for each case. The findings were categorized according to the area of the GI tract in which they were located: esophagus, stomach, small bowel (duodenum, jejunum, ileum); or colon, rectum, and other (eg, mouth). We compared the number of individual findings detected by camera 1 versus by dual-camera analysis (ie, VAJG vs PHD and JMHG) and then by camera 2 (JMRT vs PHD and $\mathrm{JMHG}$ ) to determine the level of agreement between the involved physicians and to exclude interobserver disagreement bias. We also compared the findings between camera 1 and camera 2 to determine the levels of agreement and reproducibility. An overall agreement level for all three analyses (dual-camera analysis, camera 1, and camera 2) was also calculated. Strength of agreement was classified as follows: $<0.20=$ poor; $0.21-0.40=$ fair; $0.41-0.60=$ moderate; $0.61-0.80=$ good; $0.81-1.00=$ very good

A descriptive analysis was performed using absolute values and percentages, with mean and range when appropriate. To evaluate the strength of agreement between dual-camera and single-camera observers, chance-adjusted kappa coefficients with $95 \%$ confidence intervals (CIs) were calculated for overall analysis as well as for individual GI tract areas of evaluation (esophagus, stomach, small bowel, and colon and rectum), and for specific diagnoses (eg, hiatal hernia, erosive esophagitis, diverticular disease, vascular lesions, etc).

\section{Results}

A total evaluation of the entire GI tract was possible in all patients. The quality of small-bowel and colon preparation was very good in all cases. The mean total endoscope transit time was 325 minutes (range 150-691 minutes). Mean esophageal transit time was 7.78 seconds (range 1-42 seconds), mean gastric transit time was 65.28 minutes (range 11-343 minutes), mean small bowel transit time was 131.33 minutes (range 48-254 minutes), and mean colonic transit time was 123.80 minutes (range 14-406 minutes). No difficulties in swallowing the capsule nor adverse or side effects related to the procedure were observed.

Overall, dual-camera analysis detected a total number of findings of 148, single-camera analysis with camera 1 detected 128 findings, and single-camera analysis with camera 2 detected 135 findings. Thus, dual-camera analysis provided $16 \%$ and $5 \%$ more findings compared to camera 1 and camera 2, respectively. The types of findings and their locations are summarized in Table 1.

Agreement between dual-camera analysis and camera 1 was $66 \%$ (kappa $=0.322,95 \%$ CI: 0.211-0.434), corresponding to a fair agreement. The agreement between dual-camera analysis and camera 2 was 76\% (kappa $=0.513$, 95\% CI: 0.414-0.613), corresponding to a moderate agreement. The agreement between camera 1 and camera 2 was $64 \%$ $(\mathrm{kappa}=0.278,95 \% \mathrm{CI}: 0.16-0.394)$, corresponding to a fair agreement. Overall agreement level for all three analyses (dual-camera analysis, camera 1, and camera 2), concerning all findings, was $62.7 \%$ (kappa $=0.442$, 95\% CI: $0.373-$ 0.510). Overall agreement levels (three-level agreement) according to the site of the GI tract where the findings were detected are shown in Table 2. Esophageal (kappa = 0.611), and colorectal $(\mathrm{kappa}=0.595)$ findings had the best overall level of agreement, while small bowel $($ kappa $=0.405)$ was the site with the least agreement (Table 2).

A total of 31 small-bowel angiodysplasias were detected (eleven by dual-camera analysis, nine by camera 1, and eleven by camera 2). Forty-seven colorectal polyps were detected (17 by dual-camera analysis, 15 by camera 1 , and 14 by camera 2). Most of the polyps were less than $6 \mathrm{~mm}$. Table 3 shows the overall levels of agreement for specific diseases. Perfect agreement was observed in UC and Crohn's disease $($ kappa $=1)$, where all observers reported 
Table I Whole gastrointestinal tract findings detected by each camera separately and by both cameras simultaneously

\begin{tabular}{|c|c|c|c|}
\hline Findings & $\begin{array}{l}\text { Dual analysis (both cameras) } \\
\text { (n) }\end{array}$ & $\begin{array}{l}\text { Single analysis (camera I) } \\
\text { (n) }\end{array}$ & $\begin{array}{l}\text { Single analysis (camera 2) } \\
\text { (n) }\end{array}$ \\
\hline Total number of findings & 148 & 128 & 135 \\
\hline \multicolumn{4}{|l|}{ Esophageal } \\
\hline Esophageal diverticuli & 1 & 0 & 0 \\
\hline Schatzki ring & 1 & 0 & I \\
\hline Hiatal hernia & 7 & 6 & 7 \\
\hline Erosive esophagitis & 5 & 5 & 6 \\
\hline Barrett esophagus & 2 & I & 2 \\
\hline Venous ectasia & I & 0 & 0 \\
\hline Esophageal varices & 1 & 2 & I \\
\hline Glycogenic acanthosis & 4 & 3 & 3 \\
\hline \multicolumn{4}{|l|}{ Stomach } \\
\hline Congestive gastropathy & 9 & 9 & 10 \\
\hline Gastric polyps & 4 & 4 & 3 \\
\hline Gastric angiodysplasia & I & 3 & 2 \\
\hline Bile reflux & 0 & 2 & 2 \\
\hline Gastric hyperplasia & 0 & 3 & I \\
\hline \multicolumn{4}{|l|}{ Small bowel } \\
\hline Brunner's gland hyperplasia & 1 & I & I \\
\hline Duodenitis & 3 & 4 & 3 \\
\hline Duodenum polyps & 2 & 2 & 1 \\
\hline Lymphangiectasia & 16 & 15 & 13 \\
\hline Celiac disease (atrophy) & 5 & I & 4 \\
\hline Duodenal varices & I & 0 & 0 \\
\hline Duodenal angiodysplasia & 1 & 2 & I \\
\hline Duodenum diverticuli & 0 & I & I \\
\hline Jejunal angiodysplasia & 2 & 4 & 3 \\
\hline Jejunal polyps & 2 & 2 & I \\
\hline Jejunal venous ectasia & 0 & 4 & I \\
\hline Isolated jejunal ulcers & 4 & 3 & 3 \\
\hline Jejunal xanthoma & 0 & I & 0 \\
\hline Ileal angiodysplasia & 1 & 0 & 0 \\
\hline Nodular lymphoid hyperplasia & 5 & 2 & 6 \\
\hline Ileal Crohn's disease & 2 & 2 & 2 \\
\hline NSAID enteropathy & I & 0 & 2 \\
\hline lleal cyst & 2 & I & 0 \\
\hline Ileal diverticuli & 2 & 4 & 2 \\
\hline Ileal polyp & 0 & 2 & I \\
\hline Ileal venous ectasia & 0 & 4 & 0 \\
\hline \multicolumn{4}{|l|}{ Colon and rectum } \\
\hline Diverticular disease & 17 & 18 & 16 \\
\hline Colon ulcers & 4 & 2 & 2 \\
\hline Ulcerative colitis & 2 & 2 & 2 \\
\hline Cecum angiodysplasia & 4 & 2 & 5 \\
\hline Sigmoid angiodysplasia & 3 & I & I \\
\hline Transverse angiodysplasia & 0 & I & I \\
\hline Rectal polyps & 7 & 4 & 5 \\
\hline Sigmoid polyps & 5 & 8 & 6 \\
\hline Right colon polyps & 2 & 3 & 2 \\
\hline Transverse colon polyps & 1 & 2 & 2 \\
\hline Sigmoid malignancies & 2 & I & I \\
\hline Rectal ulcer & I & I & I \\
\hline Proctitis & 0 & I & 0 \\
\hline Hemorrhoids & 13 & 5 & 8 \\
\hline \multicolumn{4}{|l|}{ Other } \\
\hline Geographic tongue & 1 & 0 & 0 \\
\hline Soft palate erythema & 0 & 2 & 0 \\
\hline
\end{tabular}

Abbreviation: NSAID, nonsteroidal anti-inflammatory drug. 
Table 2 Overall agreement (three levels) according to different sections of the gastrointestinal tract

\begin{tabular}{|c|c|c|c|c|c|}
\hline Site of findings & $\begin{array}{l}\text { Dual analysis } \\
\text { (both cameras) } \\
\text { (n) }\end{array}$ & $\begin{array}{l}\text { Single analysis } \\
\text { (camera I) } \\
\text { (n) }\end{array}$ & $\begin{array}{l}\text { Single analysis } \\
\text { (camera 2) } \\
\text { (n) }\end{array}$ & $\begin{array}{l}\text { Level of } \\
\text { agreement }\end{array}$ & $\begin{array}{l}\text { Kappa } \\
(95 \% \text { CI) }\end{array}$ \\
\hline Esophagus & 22 & 17 & 20 & $0.74 I$ & $\begin{array}{l}0.611 \\
(0.43-0.792)\end{array}$ \\
\hline Stomach & 14 & 21 & 18 & 0.673 & $\begin{array}{l}0.510 \\
(0.319-0.701)\end{array}$ \\
\hline Small bowel & 50 & 55 & 45 & 0.605 & $\begin{array}{l}0.405 \\
(0.291-0.520)\end{array}$ \\
\hline Colon and rectum & 61 & 51 & 52 & 0.730 & $\begin{array}{l}0.595 \\
(0.48-0.709)\end{array}$ \\
\hline
\end{tabular}

similar findings in all cases (Figure 1). A good agreement was observed for hiatal hernia (kappa $=0.851)$, erosive esophagitis (kappa $=0.812$ ), and diverticular disease of the colon (kappa $=0.812)$. The lowest levels of agreement were observed in small-bowel diseases such as angiodysplasias $($ kappa $=0.375)$, and nonsteroidal anti-inflammatory drug $($ NSAID) enteropathy $($ kappa $=0.372)($ Figure 1$)$.

\section{Discussion}

According to the European Society of Gastrointestinal Endoscopy (ESGE), CCE is considered a feasible and safe technique that seems to be accurate when used in average-risk individuals, may be cost-effective in screening for colorectal cancer compared with colonoscopy, and represents a useful tool for visualization of the colonic mucosa in patients with incomplete colonoscopy. ${ }^{21}$

Information regarding the use of small-bowel capsule endoscopy and CCE beyond its indications is scarce.

Table 3 Overall agreement (three levels) according to specific diagnoses among observers

\begin{tabular}{|c|c|c|c|}
\hline Disease & $\begin{array}{l}\text { Level of } \\
\text { agreement }\end{array}$ & Kappa & $95 \% \mathrm{Cl}$ \\
\hline Crohn's disease & 1.0 & 1.0 & - \\
\hline Ulcerative colitis & 1.0 & 1.0 & - \\
\hline Hiatal hernia & 0.90 & $0.85 I$ & $0.54-1.156$ \\
\hline Erosive esophagitis & 0.875 & 0.812 & $0.465-1.159$ \\
\hline Diverticular disease of the colon & 0.875 & 0.812 & $0.612-1.012$ \\
\hline Lymphangiectasia & 0.864 & 0.795 & $0.585-1.004$ \\
\hline Hemorrhoids & 0.786 & 0.643 & $0.369-0.916$ \\
\hline Gastropathy & $0.7 \mid 4$ & 0.571 & $0.309-0.832$ \\
\hline Celiac disease & 0.667 & 0.500 & $0.175-0.825$ \\
\hline Colorectal polyps & 0.660 & 0.491 & $0.290-0.691$ \\
\hline \multicolumn{4}{|l|}{ Nodular lymphoid } \\
\hline hyperplasia & 0.615 & 0.393 & $0.00 I-0.785$ \\
\hline NSAID enteropathy & 0.600 & 0.375 & $-2.64-1.014$ \\
\hline $\begin{array}{l}\text { Small-bowel } \\
\text { angiodysplasias }\end{array}$ & 0.58 & 0.372 & $0.126-0.618$ \\
\hline
\end{tabular}

Abbreviation: NSAID, nonsteroidal anti-inflammatory drug.
Kobayashi et al, ${ }^{24}$ in a study of 55 patients with OGIB, evaluated the diagnostic yield of small-bowel capsule endoscopy for gastric diseases, and found that this technique has, for diffuse lesions, sensitivity and specificity of $70 \%$ and $82 \%$, respectively. However, for localized lesions, sensitivity and specificity were $28 \%$ and $63 \%$, respectively. In another study, Rana et $\mathrm{al}^{25}$ found that up to $10 \%$ of 87 subjects evaluated with the small-bowel capsule had colonic abnormalities (including angiodysplasias, neoplasias, polyps, and Crohn's disease), suggesting that, along with appropriate preparation, the colon should also be carefully evaluated in patients undergoing smallbowel capsule endoscopy. Almeida et al, ${ }^{23}$ with the opposite approach to Rana et al's study, found that double-camera enteroscopy using CCE increases the rate of detection for smallbowel abnormalities. Although Almeida et $\mathrm{al}^{23}$ first described the use of CCE to perform a double-camera enteroscopy, this

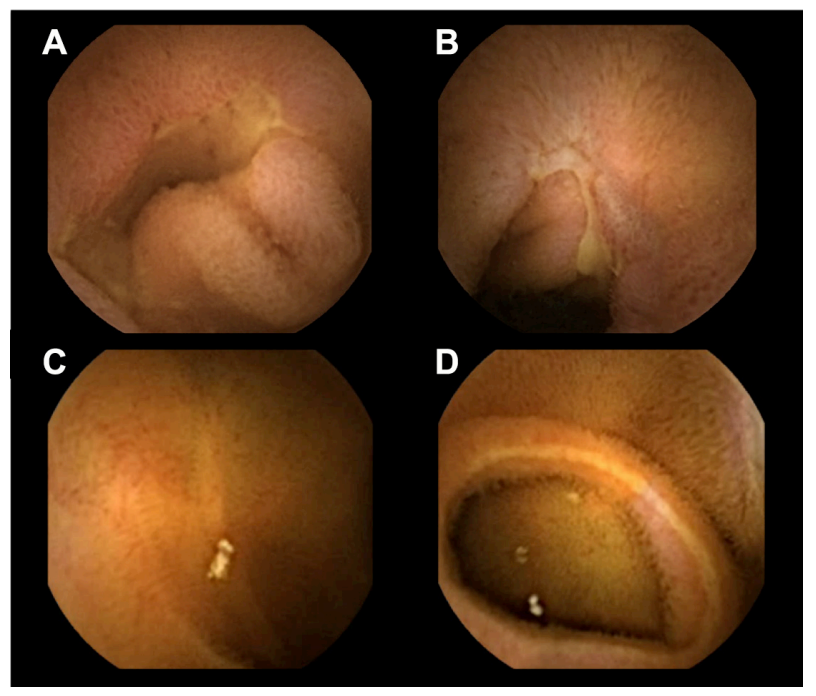

Figure I Small-bowel images obtained with capsule endoscopy of the colon. Notes: Images of a patient with ileal Crohn's disease obtained with camera I (A) and camera 2 (B). In this case, the two cameras showed the presence of the disease. Images of a patient with nonsteroidal anti-inflammatory drug enteropathy obtained with camera I (C) and camera 2 (D). In this case, only camera 2 revealed the presence of a circumferential ulceration. 
study included only ten patients and the aim was solely to evaluate the small bowel.

Following a protocol similar to that used by Almeida et al, ${ }^{23}$ we evaluated the whole GI tract; to our knowledge, this was the first study to do so. The entire GI tract evaluation was feasible because the double-camera capsule was swallowed after it restarted from the sleep mode. Thus, CCE may be used as a noninvasive, all-in-one technique to visualize the entire GI tract from the esophagus to the colon and rectum.

A major benefit of the double camera is that it significantly increases the area able to be evaluated, but also, as shown in our study, both cameras capture different sets of images that are not mutually reproducible (kappa between camera 1 and camera 2 was 0.278 ). Thus, a dual and simultaneous analysis of both cameras allows a more precise assessment of the GI tract. Although the overall agreement between observers was between fair and moderate for the entire GI evaluation, the dual-camera analysis detected between 5\% and 16\% more abnormalities compared to single-camera analysis. Previous studies have estimated that the miss rate for single-capsule endoscopy ranges between $11 \%$ and 19\%. ${ }^{2,26}$ Problems such as the potential to miss a single-mass lesion and poor visualization of some segments of the small bowel are less likely to occur if a CCE is used.

Interestingly, we found that, for some areas of the GI tract and for some diseases, the overall concordance was moderate to good. For example, kappa for overall esophageal findings, erosive esophagitis, and hiatal hernia were 0.611, 0.51 , and 0.812 , respectively. Similar results have been previously reported with kappa values ranging from 0.42 to 0.67 for esophagitis. ${ }^{12,27}$ In contrast, the use of esophageal capsule endoscopy demonstrates moderate sensitivity and specificity for the diagnosis of Barrett's esophagus. In our study, the number of patients with Barrett's esophagus was small $(n=2)$; thus, we cannot assess the agreement for this condition.

Small-bowel capsule endoscopy provides a high diagnostic yield for small-bowel mucosal lesions, and its use is recommended in specific scenarios of IBD, such as in the pediatric population, where it may help lead to a definitive diagnosis and sometimes can result in reclassification of $\mathrm{UC} /$ indeterminate colitis to Crohn's disease. ${ }^{28-30}$ To date, the role of CCE in IBD has been evaluated in only one case series. ${ }^{22}$ Specifically, CCE has been compared with colonoscopy with the aim of evaluating its accuracy in monitoring colonic inflammation in patients with suspected or known UC. In this preliminary experience, CCE yielded encouraging results for detecting active ulcerative colitis (ie, sensitivity $77 \%$, specificity $78 \%$ ) and good agreement with colonoscopy. Although we had a small sample size, we found that, for detecting IBD, CCE is a good method with a high level of agreement.

Regarding other colorectal findings, a good agreement level was found for diverticular disease of the colon. To our knowledge, ours is the first study to report on this disease. It is essential to remark that, in all cases, diverticular disease was considered non-complicated. Further studies are required to analyze diagnostic accuracy and clinical use of CCE for diverticular disease of the colon. We found 47 polyps, most of which were small. As reported by others, most colonic polyps discovered at screening are diminutive, with negligible risk of harboring advanced features (highgrade dysplasia, villous component, or malignancy). ${ }^{20,31}$ Moreover, $40 \%$ of diminutive colonic polyps are hyperplastic rather than adenomatous. ${ }^{32}$ Diminutive lesions identified by a noninvasive test may also be missed by colonoscopy, because of the imperfect sensitivity of the latter for diminutive lesions. ${ }^{33}$

It is also important to remark that, in up to $62 \%(13 / 21)$ of our subjects, internal hemorrhoids were detected by CCE. This finding makes obvious that a bowel preparation using booster doses of $30 \mathrm{~mL}$ sodium phosphate solution can guarantee that the capsule reaches the rectum, allowing a complete GI evaluation.

Comparing single-camera analysis and dual-camera analysis, the lower level of agreement was observed in smallbowel diseases such as angiodysplasias (kappa $=0.375)$, and NSAID enteropathy (kappa $=0.372$ ). This finding could be explained because single-camera analysis has limited vision and each camera may capture different, non-reproducible findings. Thus, according to our results, as in the Almeida study, ${ }^{23}$ a double-camera enteroscopy using CCE is a better option for complete evaluation of small-bowel diseases.

There are some limitations in our study that should be acknowledged. First of all, the study had a small sample size and the CCE was used in different diseases. Also, we did not evaluate the upper part of the GI tract, the small bowel, or the colon with other invasive techniques (such as endoscopy, enteroscopy, and colonoscopy), but we must say that this was beyond our aim. Further studies are required to compare findings detected by CCE in the whole GI tract versus those detected with standard techniques. The statistical agreement analysis was calculated for overall findings and for some lesions because there were not enough subjects to do a perpatient analysis. Also, because each patient had more than one abnormality detected by CCE, an analysis per patient was extremely difficult to perform. 
Although we have shown, using CCE, that more findings could be detected in the colon and other parts of the GI tract, this technique does not exclude false negative results at all. For example, a CCE examination may be incomplete, mainly because of a slow GI transit that may mean that the capsule stays longer periods in some segments of the GI tract. Therefore, some segments may not be visualized by CCE. In addition, although we describe that an entire GI tract evaluation was possible in all cases, we must acknowledge that some parts of the stomach, specifically the fundus, are not able to be assessed using capsule endoscopy.

\section{Conclusion}

The use of a dual-camera analysis using CCE for the evaluation of the GI tract is feasible and detects more abnormalities when compared with a single-camera analysis. However, further studies are required that include larger sample sizes, specific populations (eg, obscure bleeding), and a costeffectiveness analysis.

\section{Acknowledgments}

The authors wish to thank Mr Eli de la Cruz Patiño and $\mathrm{Mr}$ Job Ulises Reyes Huerta for their technical support.

\section{Disclosure}

The authors report no conflicts of interest in this work.

\section{References}

1. Delvaux M, Gay G. International Conference on Capsule and DoubleBalloon Endoscopy (ICCD). Paris, August 27-28, 2010. Endoscopy. 2011;43(6):533-539.

2. Leighton JA. The role of endoscopic imaging of the small bowel in clinical practice. Am J Gastroenterol. 2011;106(1):27-36.

3. Triester SL, Leighton JA, Leontiadis GI, et al. A meta-analysis of the yield of capsule endoscopy compared to other diagnostic modalities in patients with obscure gastrointestinal bleeding. Am J Gastroenterol. $2005 ; 100: 2407-2418$.

4. Liu K, Kaffes AJ. Review article: the diagnosis and investigation of obscure gastrointestinal bleeding. Aliment Pharmacol Ther. 2011;34(4):416-423.

5. Rokkas T, Niv Y. The role of video capsule endoscopy in the diagnosis of celiac disease: a meta-analysis. Eur J Gastroenterol Hepatol. 2012;24(3): 303-308.

6. Günther U, Bojarski C, Buhr HJ, Zeitz M, Heller F. Capsule endoscopy in small-bowel surveillance of patients with hereditary polyposis syndromes. Int J Colorectal Dis. 2010;25(11):1377-1382.

7. Postgate AJ, Will OC, Fraser CH, Fitzpatrick A, Phillips RK, Clark SK. Capsule endoscopy for the small bowel in juvenile polyposis syndrome: a case series. Endoscopy. 2009;41(11):1001-1004.

8. Bailey AA, Debinski HS, Appleyard MN, et al. Diagnosis and outcome of small bowel tumors found by capsule endoscopy: a threecenter Australian experience. Am J Gastroenterol. 2006;101(10): 2237-2243.

9. Schwartz GD, Barkin JS. Small-bowel tumors detected by wireless capsule endoscopy. Dig Dis Sci. 2007;52:1026-1030.
10. Caunedo A, Rodríguez-Téllez M, García-Montes JM, et al. Usefulness of capsule endoscopy in patients with suspected small bowel disease. Rev Esp Enferm Dig. 2004;6(1):10-21. English, Spanish.

11. Niv Y. Capsule endoscopy: no longer limited to the small bowel. Isr Med Assoc J. 2010;12(3):178-180.

12. Galmiche JP, Sacher-Huvelin S, Coron E, et al. Screening for esophagitis and Barrett's esophagus with wireless esophageal capsule endoscopy: a multicenter prospective trial in patients with reflux symptoms. $\mathrm{Am} \mathrm{J}$ Gastroenterol. 2008;103:538-545.

13. Eliakim R, Yassin K, Shlomi I, Suissa A, Eisen GM. A novel diagnostic tool for detecting oesophageal pathology: the PillCam oesophageal video capsule. Aliment Pharmacol Ther. 2004;20(10):1083-1089.

14. Fernandez-Urien I, Carretero C, Armendariz R, Muñoz-Navas M. Esophageal capsule endoscopy. World J Gastroenterol. 2008;14(34): 5254-5260

15. Guturu P, Sagi SV, Ahn D, Jaganmohan S, Kuo YF, Sood GK. Capsule endoscopy with PILLCAM ESO for detecting esophageal varices: a meta-analysis. Minerva Gastroenterol Dietol. 2011;57(1):1-11.

16. Eliakim R, Fireman Z, Gralnek IM, et al. Evaluation of the PillCam Colon capsule in the detection of colonic pathology: results of the first multicenter, prospective, comparative study. Endoscopy. 2006;38(10): 963-970.

17. Herrerías-Gutiérrez JM, Argüelles-Arias F, Caunedo-Álvarez A, et al. PillCamColon Capsule for the study of colonic pathology in clinical practice. Study of agreement with colonoscopy. Rev Esp Enferm Dig. 2011;103(2):69-75.

18. Schoofs N, Devière J, Van Gossum A. PillCam colon capsule endoscopy compared with colonoscopy for colorectal tumor diagnosis: a prospective pilot study. Endoscopy. 2006;38:971-977.

19. Van Gossum A, Munoz-Navas M, Fernandez-Urien I, et al. Capsule endoscopy versus colonoscopy for the detection of polyps and cancer. N Engl J Med. 2009;361:264-270.

20. Hassan C, Zullo A, Winn S, Morini S. Cost-effectiveness of capsule endoscopy in screening for colorectal cancer. Endoscopy. 2008;40: 414-421.

21. Spada C, Hassan C, Galmiche JP, et al; European Society of Gastrointestinal Endoscopy. Colon capsule endoscopy: European Society of Gastrointestinal Endoscopy (ESGE) Guideline. Endoscopy. 2012;44(5): 527-536.

22. Sung JJY, Ching JY, Leung WK, et al. Assessment of colonic inflammatory lesions and ulcerative colitis with PillCam Colon capsule endoscopy compared to colonoscopy. Endoscopy. 2008;40:A199.

23. Almeida N, Figuereido P, Lopes S, et al. Is it the end of single camera small bowel capsule as we know it? Presented at the 16th European Gastroenterology Week "UEGW;" October 18-22;2008; Vienna, Austria. OP 144

24. Kobayashi Y, Watabe H, Yamada A, et al. Diagnostic yield of capsule endoscopy for gastric diseases. Abdom Imaging. 2012;37(1):29-34

25. Rana SS, Bhasin DK, Singh K. Colonic lesions in patients undergoing small bowel capsule endoscopy. Int J Colorectal Dis. 2011;26(6): 699-702.

26. Rondonotti E, Villa F, Mulder CJ, Jacobs MA, de Franchis R. Small bowel capsule endoscopy in 2007: indications, risks and limitations. World J Gastroenterol. 2007;13:6140-6149.

27. Delvaux M, Papanikolaou IS, Fassler I, et al. Esophageal capsule endoscopy in patients with suspected esophageal disease: double blinded comparison with esophagogastroduodenoscopy and assessment of interobserver variability. Endoscopy. 2008;40(1):16-22.

28. Herrerías JM, Caunedo A, Rodríguez-Téllez M, Pellicer F, Herrerías JM Jr. Capsule endoscopy in patients with suspected Crohn's disease and negative endoscopy. Endoscopy. 2003;35:564-568.

29. Marmo R, Rotondano G, Piscopo R, et al. Capsule endoscopy versus enteroclysis in the detection of small-bowel involvement in Crohn's disease: a prospective trial. Clin Gastroenterol Hepatol. 2005;3: 772-776.

30. Gralnek IM, Cohen SA, Ephrath H, et al. Small bowel capsule endoscopy impacts diagnosis and management of pediatric inflammatory bowel disease: a prospective study. Dig Dis Sci. 2012;57(2):465-471. 
31. Lieberman D, Moravec M, Holub J, Michaels L, Eisen G. Polyp size and advanced histology in patients undergoing colonoscopy screening: implications for CT colonography. Gastroenterology. 2008;135:1100-1105.

32. Weston AP, Campbell DR. Diminutive colonic polyps: histopathology, spatial distribution, concomitant significant lesions, and treatment complications. Am J Gastroenterol. 1995;90:24-28.
33. van Rijn JC, Reitsma JB, Stoker J, Bossuyt PM, van Deventer SJ, Dekker E. Polyp miss rate determined by tandem colonoscopy: a systematic review. Am J Gastroenterol. 2006;10:343-350.

\section{Publish your work in this journal}

Clinical and Experimental Gastroenterology is an international, peerreviewed, open access journal, publishing all aspects of gastroenterology in the clinic and laboratory, including: Pathology, pathophysiology of gastrointestinal disease; Investigation and treatment of gastointestinal disease; Pharmacology of drugs used in the alimentary tract;
Immunology/genetics/genomics related to gastrointestinal disease. This journal is indexed on CAS. The manuscript management system is completely online and includes a very quick and fair peer-review system. Visit http://www.dovepress.com/testimonials.php to read real quotes from published authors.

Submit your manuscript here: http://www.dovepress.com/clinical-and-experimental-gastroenterology-journal 